\title{
Gelfand numbers and metric entropy of convex hulls in Hilbert spaces
}

\author{
by \\ Bernd Carl (Jena) and David E. Edmunds (Brighton) \\ Dedicated to Aleksander Pelczyński on his 70th birthday
}

\begin{abstract}
For a precompact subset $K$ of a Hilbert space we prove the following inequalities:$$
n^{1 / 2} c_{n}(\operatorname{cov}(K)) \leq c_{K}\left(1+\sum_{k=1}^{n} k^{-1 / 2} e_{k}(K)\right), \quad n \in \mathbb{N},
$$

and$$
k^{1 / 2} c_{k+n}(\operatorname{cov}(K)) \leq c\left[\log ^{1 / 2}(n+1) \varepsilon_{n}(K)+\sum_{j=n+1}^{\infty} \frac{\varepsilon_{j}(K)}{j \log ^{1 / 2}(j+1)}\right],
$$

$k, n \in \mathbb{N}$, where $c_{n}(\operatorname{cov}(K))$ is the $n$th Gelfand number of the absolutely convex hull of $K$ and $\varepsilon_{k}(K)$ and $e_{k}(K)$ denote the $k$ th entropy and $k$ th dyadic entropy number of $K$, respectively. The inequalities are, essentially, a reformulation of the corresponding inequalities given in [CKP] which yield asymptotically optimal estimates of the Gelfand numbers $c_{n}(\operatorname{cov}(K))$ provided that the entropy numbers $\varepsilon_{n}(K)$ are slowly decreasing. For example, we get optimal estimates in the non-critical case where $\varepsilon_{n}(K) \preceq \log ^{-\alpha}(n+1)$, $\alpha \neq 1 / 2,0<\alpha<\infty$, as well as in the critical case where $\alpha=1 / 2$. For $\alpha=1 / 2$ we show the asymptotically optimal estimate $c_{n}(\operatorname{cov}(K)) \preceq n^{-1 / 2} \log (n+1)$, which refines the corresponding result of Gao $[\mathrm{Ga}]$ obtained for entropy numbers. Furthermore, we establish inequalities similar to that of Creutzig and Steinwart [CrSt] in the critical as well as non-critical cases. Finally, we give an alternative proof of a result by $\mathrm{Li}$ and Linde [LL] for Gelfand and entropy numbers of the absolutely convex hull of $K$ when $K$ has the shape $K=\left\{t_{1}, t_{2}, \ldots\right\}$, where $\left\|t_{n}\right\| \leq \sigma_{n}, \sigma_{n} \downarrow 0$. In particular, for $\sigma_{n} \leq \log ^{-1 / 2}(n+1)$, which corresponds to the critical case, we get a better asymptotic behaviour of Gelfand numbers, $c_{n}(\operatorname{cov}(K)) \preceq n^{-1 / 2}$.
\end{abstract}

1. Introduction and basic tools. The main aim of this paper is to complement existing results concerning the behaviour of Gelfand numbers of absolutely convex hulls $\operatorname{cov}(K)$ of precompact subsets $K$ of a Hilbert

2000 Mathematics Subject Classification: Primary 41A46, 47B06; Secondary 46B07, 46B20, $52 \mathrm{~A} 07$.

Key words and phrases: widths, metric entropy, entropy numbers, convex sets.

The first author acknowledges the support of EPSRC, grant number GR/S04895/01. 
space $H$ when the entropy numbers of $K$ decay slowly. This enables us to refine results of Gao [Ga], Creutzig and Steinwart [CrSt] in the so-called critical case. The upper estimates, which we establish in the critical case, are already implicitly contained in [CKP]. By using an ingenious example of Gao we prove that in the critical case the estimates are asymptotically optimal. Moreover, we show that there is a difference between the behaviour of Gelfand numbers of absolutely convex hulls $\operatorname{cov}(K)$ generated by sets $K \subset H$ consisting of "many" and those with "few" extremal points. For more information and references about the behaviour of the metric entropy of convex hulls we refer to the papers [C2], [CKP] and [St].

For our purposes we use the following notation and quantities. Let $(M, d)$ be a metric space and $B(s, \varepsilon):=\{t \in M \mid d(s, t) \leq \varepsilon\}$ be the closed $\varepsilon$-ball in $M$ with centre $s$. For a bounded set $K \subset M$ and $\varepsilon>0$ the covering number of $K$ is defined by

$$
N(K ; \varepsilon):=\inf \left\{n \mid \exists s_{1}, \ldots, s_{n} \in M: K \subset \bigcup_{i=1}^{n} B\left(s_{i}, \varepsilon\right)\right\} .
$$

We denote the entropy numbers of $K$ by

$$
\varepsilon_{n}(K):=\inf \{\varepsilon>0 \mid N(K ; \varepsilon) \leq n\}
$$

and its dyadic entropy numbers by

$$
e_{n}(K):=\varepsilon_{2^{n-1}}(K), \quad n \in \mathbb{N} .
$$

Moreover, the entropy numbers of a (bounded linear) operator $T: E \rightarrow F$ from a Banach space $E$ into a Banach space $F$ are defined by

$$
\varepsilon_{n}(T):=\varepsilon_{n}\left(T\left(B_{E}\right)\right)
$$

and its dyadic entropy numbers by

$$
e_{n}(T):=\varepsilon_{2^{n-1}}(T), \quad n \in \mathbb{N},
$$

where $B_{E}$ is the closed unit ball of $E$. Furthermore, the $n$th approximation, Gelfand and Kolmogorov numbers of $T$ are defined by

$$
\begin{aligned}
& a_{n}(T):=\inf \{\|T-A\| \mid \operatorname{rank}(A)<n\}, \\
& c_{n}(T):=\inf \left\{\left\|\left.T\right|_{M}\right\| \mid M \subset E, \operatorname{codim}(M)<n\right\}, \\
& d_{n}(T):=\inf \left\{\left\|Q_{N}^{F} T\right\| \mid N \subset E, \operatorname{dim} N<n\right\},
\end{aligned}
$$

respectively, where $Q_{N}^{F}: F \rightarrow F / N$ is the quotient map. We have $c_{n}(T)=$ $d_{n}\left(T^{\prime}\right)$, where $T^{\prime}$ is the dual operator of $T$.

If $l_{1}(K)$ denotes the Banach space of all summable families of real numbers $\left(\xi_{t}\right)_{t \in K}$ over the index set $K$ with the norm given by

$$
\left\|\left(\xi_{t}\right)\right\|=\sum_{t \in K}\left|\xi_{t}\right|
$$


then the entropy numbers and Gelfand numbers of the absolutely convex hull $\operatorname{cov}(K)$ of a bounded set $K \subset E$ of a Banach space $E$ are expressed in terms of entropy and Gelfand numbers of operators: $e_{n}(\operatorname{cov}(K))=e_{n}(T)$ and $c_{n}(\operatorname{cov}(K))=c_{n}(T)$, where $T: l_{1}(K) \rightarrow E$ is the operator defined on the canonical basis $\left(e_{t}\right)_{t \in K}$ of $l_{1}(K)$ by $T e_{t}:=t$.

Finally, we recall the $l$-norm of an operator $T: E \rightarrow F$ (or $\pi_{\gamma}$ summing norm in [LP]). Let $l_{2}^{n}$ be the $n$-dimensional Euclidean space and $S: l_{2}^{n} \rightarrow F$ an operator; then the $l$-norm of $S$ is defined by

$$
l(S):=\left(\int_{\mathbb{R}^{n}}\|S x\|^{2} d \gamma_{n}(x)\right)^{1 / 2},
$$

where $\gamma_{n}$ is the canonical Gaussian probability measure of $\mathbb{R}^{n}$; and for an operator $T: E \rightarrow F$ we define

$$
l(T):=\sup \left\{l(T A) \mid\left\|A: l_{2}^{n} \rightarrow E\right\| \leq 1, n \in \mathbb{N}\right\} .
$$

If $A: E_{0} \rightarrow E$ and $B: F \rightarrow F_{0}$ are operators between Banach spaces, then $l$ has the ideal property (cf. $[\mathrm{LP}])$,

$$
l(B T A) \leq\|B\| l(T)\|A\| .
$$

Now we give diverse tools for estimating Gelfand and entropy numbers of absolutely convex hulls in Hilbert spaces. We start with two general inequalities of $[\mathrm{CKP}]$.

THEOREM A. There is a universal constant $c>0$ such that for each precompact subset $K$ of the unit ball $B_{H}$ of a Hilbert space $H$ and for all $n \in \mathbb{N}$ we have

$$
n^{1 / 2} c_{n}(\operatorname{cov}(K)) \leq c \inf _{\varepsilon>0}\left\{\int_{\varepsilon / 4}^{1} \log ^{1 / 2}(N(K ; s)) d s+n^{1 / 2} \varepsilon\right\} .
$$

(ii) There is a universal constant $c>0$ such that for each precompact subset $K$ of a Hilbert space $H$ and for all $k, n \in \mathbb{N}$ we have

$$
k^{1 / 2} c_{k+n}(\operatorname{cov}(K)) \leq c \int_{0}^{\varepsilon_{n}(K)} \log ^{1 / 2}(N(K ; s)) d s .
$$

The next theorem is a reformulation of Theorem A.

Theorem B. Let $K$ be a precompact subset of a Hilbert space $H$. Then the following inequalities hold:

$$
n^{1 / 2} c_{n}(\operatorname{cov}(K)) \leq c_{K}\left(1+\sum_{k=1}^{n} k^{-1 / 2} e_{k}(K)\right) \quad \text { for } n \in \mathbb{N},
$$


where $c_{K} \leq c\left(1+\sup _{t \in K}\|t\|\right)$ and $c>0$ is an absolute constant;

(ii) $k^{1 / 2} c_{k+n}(\operatorname{cov}(K)) \leq c\left[\log ^{1 / 2}(n+1) \varepsilon_{n}(K)+\sum_{j=n+1}^{\infty} \frac{\varepsilon_{j}(K)}{j \log ^{1 / 2}(j+1)}\right]$

for $k, n \in \mathbb{N}$, where $c>0$ is an absolute constant.

Proof. (i) First we assume that $K$ is contained in $B_{H}$. By Theorem A we get, with an absolute constant $c>0$,

$$
n^{1 / 2} c_{n}(\operatorname{cov}(K)) \leq c\left(\int_{\varepsilon / 4}^{1} \log _{2}^{1 / 2}(N(K ; s)) d s+n^{1 / 2} \varepsilon\right)
$$

for $n \in \mathbb{N}$ and $\varepsilon>0$.

We suppose $e_{n}(K)>0$; the case $e_{n}(K)=0$ can be treated more easily. Put $\varepsilon:=4 e_{n}(K)$. Then we have, with $e_{0}(K):=1$,

$$
\begin{aligned}
\int_{e_{n}(K)}^{1} \log _{2}^{1 / 2}(N(K ; s)) d s & =\sum_{k=1}^{n} \int_{e_{k}(K)}^{e_{k-1}(K)} \log _{2}^{1 / 2}(N(K ; s)) d s \\
& \leq \sum_{k=1}^{n} \int_{e_{k}(K)}^{e_{k-1}(K)} k^{1 / 2} d s=\sum_{k=1}^{n} k^{1 / 2}\left(e_{k-1}(K)-e_{k}(K)\right) \\
& =1+\sum_{k=1}^{n-1}\left((k+1)^{1 / 2}-k^{1 / 2}\right) e_{k}(K)-n^{1 / 2} e_{n}(K) \\
& \leq 1+\frac{1}{2} \sum_{k=1}^{n} k^{-1 / 2} e_{k}(K)-n^{1 / 2} e_{n}(K)
\end{aligned}
$$

Thus

$$
\begin{aligned}
\int_{e_{k}(K)}^{1} \log _{2}^{1 / 2}(N(K ; s)) d s+4 n^{1 / 2} & e_{n}(K) \\
& \leq 1+\frac{1}{2} \sum_{k=1}^{n-1} k^{-1 / 2} e_{k}(K)+3 n^{1 / 2} e_{n}(K) \\
& \leq 1+\frac{7}{2} \sum_{k=1}^{n} k^{-1 / 2} e_{k}(K) .
\end{aligned}
$$

This implies, with an absolute constant $c>0$, the desired inequality for $K \subset B_{H}$ :

$$
n^{1 / 2} c_{n}(\operatorname{cov}(K)) \leq c\left(1+\sum_{k=1}^{n} k^{-1 / 2} e_{k}(K)\right)
$$


If $K \subset H$ is a precompact set with $d:=\sup _{t \in K}\|t\| \geq 1$, then by the previous inequality and the equalities

$$
c_{n}(\operatorname{cov}(K))=d c_{n}(\operatorname{cov}(B)), \quad e_{n}(K)=d e_{n}(B),
$$

where $B:=\{t / d \mid t \in K\} \subset B_{H}$, we get the estimate

$$
n^{1 / 2} c_{n}(\operatorname{cov}(K)) \leq c d\left(1+\sum_{k=1}^{n} k^{-1 / 2} e_{k}(K)\right) .
$$

Combining (1) and (2) we obtain inequality (i) of the theorem with $c_{K} \leq$ $c\left(1+\sup _{t \in K}\|t\|\right)$.

(ii) This time the starting point is inequality (ii) of Theorem A for a precompact set $K \subset H$ :

$$
k^{1 / 2} c_{k+n}(\operatorname{cov}(K)) \leq c \int_{0}^{\varepsilon_{n}(K)} \log ^{1 / 2}(N(K ; s)) d s .
$$

Indeed, the right-hand side of the inequality can be estimated as follows:

$$
\begin{aligned}
\int_{0}^{\varepsilon_{n}(K)} \log ^{1 / 2}(N(K ; s)) d s=\sum_{j=n}^{\infty} \int_{\varepsilon_{j+1}(K)}^{\varepsilon_{j}(K)} \log ^{1 / 2}(N(K ; s)) d s \\
\leq \sum_{j=n}^{\infty} \log ^{1 / 2}\left(N\left(K, \varepsilon_{j+1}(K)\right)\right)\left(\varepsilon_{j}(K)-\varepsilon_{j+1}(K)\right) \\
\leq \sum_{j=n}^{\infty} \log ^{1 / 2}(j+2)\left(\varepsilon_{j}(K)-\varepsilon_{j+1}(K)\right) \\
=\log ^{1 / 2}(n+2) \varepsilon_{n}(K)+\sum_{j=n+1}^{\infty}\left(\log ^{1 / 2}(j+2)-\log ^{1 / 2}(j+1)\right) \varepsilon_{j}(K) \\
\leq \log ^{1 / 2}(n+2) \varepsilon_{n}(K)+\frac{1}{2} \sum_{j=n+1}^{\infty} \frac{\varepsilon_{j}(K)}{(j+1) \log ^{1 / 2}(j+1)}
\end{aligned}
$$

This estimate yields the desired inequality (ii).

The following inequality of Theorem 1.3 in $[\mathrm{CKP}]$ is a version of the corresponding inequality in [C1].

THEOREM C. Let $\left(s_{n}\right)$ be a positive and increasing sequence with the property that there exists a constant $\gamma \geq 1$ such that $s_{2 n} \leq \gamma s_{n}$ for all $n \in \mathbb{N}$. Then there exists a constant $c_{\gamma} \geq 1$ such that for all operators $T: E \rightarrow F$ between Banach spaces $E, F$ and all $n \in \mathbb{N}$,

$$
\sup _{1 \leq k \leq n} s_{k} e_{k}(T) \leq c_{\gamma} \sup _{1 \leq k \leq n} s_{k} c_{k}(T) .
$$


Finally, we need a refined version of the Sudakov-type inequality due to A. Pajor and N. Tomczak-Jaegermann.

TheOREM D ([PT]). There is a constant $c \geq 1$ such that for all operators $T: E \rightarrow H$ from a Banach space $E$ into a Hilbert space $H$ and all $n \in \mathbb{N}$,

$$
n^{1 / 2} c_{n}(T) \leq c l\left(T^{\prime}\right) .
$$

By Gordon [Go] we know that $c \leq \sqrt{2}$.

2. Results. In this section we give several propositions, which will be proved in Section 3. The first result refines an inequality by Creutzig and Steinwart $[\mathrm{CrSt}]$ in the critical case which was originally given in terms of entropy numbers of absolutely convex hulls.

Proposition 1. Let $-\infty<\beta<1$ and let $K$ be a precompact subset of a Hilbert space $H$. Then for all $n \in \mathbb{N}$,

$$
\sup _{1 \leq k \leq n} k^{1 / 2} \log ^{\beta-1}(k+1) c_{k}(\operatorname{cov}(K)) \leq c_{K, \beta}\left(1+\sup _{1 \leq k \leq n} k^{1 / 2} \log ^{\beta}(k+1) e_{k}(K)\right),
$$

where

$$
c_{K, \beta} \leq c\left(1+\frac{1}{1-\beta}\right)\left(1+\sup _{t \in K}\|t\|\right)
$$

and $c>0$ is an absolute constant.

Moreover, by using the basic tools we also get the following result of Steinwart $[\mathrm{St}]$.

Proposition 2. Let $0<\alpha<1 / 2$ and $K$ be a precompact subset of a Hilbert space $H$. Then for all $n \in \mathbb{N}$,

$$
\sup _{1 \leq k \leq n} k^{\alpha} c_{k}(\operatorname{cov}(K)) \leq c_{K, \alpha}\left(1+\sup _{1 \leq k \leq n} k^{\alpha} e_{k}(K)\right)
$$

where

$$
c_{K, \alpha} \leq \frac{c}{1-2 \alpha}\left(1+\sup _{t \in K}\|t\|\right)
$$

and $c>0$ is an absolute constant.

The next result gives the asymptotic behaviour of Gelfand numbers of absolutely convex hulls, which in the non-critical case $0<\alpha<\infty, \alpha \neq 1 / 2$ can already be found in [CKP].

Proposition 3. Let $0<\alpha<\infty,-\infty<\beta<\infty$ and let $K$ be a precompact subset of a Hilbert space $H$. If $e_{n}(K) \preceq n^{-\alpha} \log ^{-\beta}(n+1)$, then we have, in the non-critical case $\alpha \neq 1 / 2$,

$$
c_{n}(\operatorname{cov}(K)) \preceq\left\{\begin{array}{r}
n^{-\alpha} \log ^{-\beta}(n+1) \quad \text { for } 0<\alpha<1 / 2,-\infty<\beta<\infty, \\
n^{-1 / 2} \log ^{1 / 2-\alpha}(n+1) \log ^{-\beta}(\log (n+1)+1) \\
\text { for } 1 / 2<\alpha<\infty,-\infty<\beta<\infty,
\end{array}\right.
$$


and in the critical case $\alpha=1 / 2,-\infty<\beta<1$,

$$
c_{n}(\operatorname{cov}(K)) \preceq n^{-1 / 2} \log ^{1-\beta}(n+1) .
$$

The estimates are asymptotically optimal.

REMARK. From the estimates of the previous proposition it is interesting to see that the asymptotic behaviour of Gelfand numbers of absolutely convex hulls has a sudden jump if $\alpha$ crosses the point $1 / 2$. This is why we call $\alpha=1 / 2$ the critical case.

Finally we turn to Gelfand numbers of absolutely convex hulls generated by "few" extremal points. In [LL], Li and Linde studied the Gelfand numbers and metric entropy of $\operatorname{cov}(K)$ via certain quantities originating in the theory of majorizing measures. Among other results, they obtained some finer estimates of $c_{n}(\operatorname{cov}(K))$ for absolutely convex hulls generated by few extremal points which lead to sharper results in the critical case $\alpha=1 / 2$. The result is stated in the next proposition. Moreover, we are going to give an alternative proof of it.

Proposition 4. Let $K=\left\{t_{1}, t_{2}, \ldots\right\} \subset H$ be a precompact set such that $\left\|t_{n}\right\| \leq \sigma_{n}$, where $\sigma_{1} \geq \sigma_{2} \geq \ldots \geq 0$ and $\lim _{n \rightarrow \infty} \sigma_{n}=0$. Then the following estimates hold:

(i) If $\log ^{1 / 2}(n+1) \sigma_{n}$ is decreasing, then for all $n \in \mathbb{N}$,

$$
n^{1 / 2} c_{2 n-1}(\operatorname{cov}(K)) \leq c \log ^{1 / 2}(n+1) \sigma_{n},
$$

where $c>0$ is an absolute constant.

(ii) If $\log ^{1 / 2}(n+1) \sigma_{n}$ is increasing, then for all $n \in \mathbb{N}$,

$$
c_{n}(\operatorname{cov}(K)) \leq c \sigma_{2^{n}}
$$

where $c>0$ is an absolute constant.

The estimates are asymptotically optimal for slowly decreasing sequences $\left(\sigma_{n}\right)$. In particular, for $\sigma_{n} \preceq \log ^{-\alpha}(n+1), 0<\alpha<\infty$, we have

$$
c_{n}(\operatorname{cov}(K)) \preceq \begin{cases}n^{-\alpha} & \text { for } 0<\alpha<1 / 2, \\ n^{-1 / 2} \log ^{1 / 2-\alpha}(n+1) & \text { for } 1 / 2 \leq \alpha<\infty .\end{cases}
$$

From the previous estimate we see that in the case $\alpha=1 / 2$ we have

$$
e_{n}(K) \preceq n^{-1 / 2} \quad \text { and } \quad c_{n}(\operatorname{cov}(K)) \preceq n^{-1 / 2} .
$$

If we compare this result with the general result of Proposition 3 for $\alpha=1 / 2$ and $\beta=0$, then we observe that the additional logarithmic term does not appear. So we have in the critical case a difference between the asymptotic behaviour of Gelfand numbers of absolutely convex hulls although the (dyadic) entropy numbers have the same asymptotic behaviour: $e_{n}(K) \preceq n^{-1 / 2}$. This phenomenon depends on the fact that the precompact set $K$ either contains "many" extremal or "few" extremal points. 
Concluding Remarks. (a) From the inequality of Theorem $\mathrm{C}$ we see that the estimates of Propositions 1-3 remain valid for the dyadic entropy numbers $e_{n}(\operatorname{cov}(K))$ instead of the Gelfand numbers $c_{n}(\operatorname{cov}(K))$ of the absolutely convex hull of $K$. In particular, for a precompact subset $K$ of a Hilbert space $H$ we recover the estimate of Gao [Ga],

$$
e_{n}(\operatorname{cov}(K)) \preceq n^{-1 / 2} \log (n+1) \quad \text { for } e_{n}(K) \preceq n^{-1 / 2} .
$$

(b) Moreover, from the proof of Proposition 6.4 in [CKP] we can also conclude that for a precompact subset $K$ of a Banach space $E$ of type $p$ the asymptotic estimate

$$
e_{n}(\operatorname{cov}(K)) \preceq n^{-(1-1 / p)} \log (n+1) \quad \text { for } e_{n}(K) \preceq n^{-(1-1 / p)}
$$

is valid. This estimate is the critical case for a Banach space of type $p$. It has been recently obtained by Creutzig and Steinwart in a more general setting [CrSt]. They also showed that this estimate is asymptotically optimal, thus extending the Hilbert space result of Gao [Ga].

\section{Proofs of the results}

Proof of Proposition 1. The inequality of Proposition 1 is an easy consequence of inequality (i) of Theorem B. Indeed, if $\beta<1$ the right-hand side of (i) can be estimated by

$$
\begin{aligned}
\sum_{k=1}^{n} k^{-1 / 2} e_{k}(K) & \leq\left(\sum_{k=1}^{n} k^{-1} \log ^{-\beta}(k+1)\right) \sup _{1 \leq k \leq n} k^{1 / 2} \log ^{\beta}(k+1) e_{k}(K) \\
& \leq 2 \int_{0}^{n} \frac{d s}{(s+1) \log ^{\beta}(s+1)} \sup _{1 \leq k \leq n} k^{1 / 2} \log ^{\beta}(k+1) e_{k}(K) \\
& \leq \frac{2}{1-\beta} \log ^{1-\beta}(n+1) \sup _{1 \leq k \leq n} k^{1 / 2} \log ^{\beta}(k+1) e_{k}(K) .
\end{aligned}
$$

Thus, by (i) of Theorem B we get the estimate

$n^{1 / 2} c_{n}(\operatorname{cov}(K)) \leq c_{K}\left(1+\frac{2}{1-\beta} \log ^{1-\beta}(n+1) \sup _{1 \leq k \leq n} k^{1 / 2} \log (k+1) e_{n}(K)\right)$, yielding the inequality

$$
n^{1 / 2} \log ^{\beta-1}(n+1) c_{n}(\operatorname{cov}(K)) \leq c_{K, \beta}\left(1+\sup _{1 \leq k \leq n} k^{1 / 2} \log ^{\beta}(k+1) e_{k}(K)\right)
$$

for $n \in \mathbb{N}$, where

$$
c_{K, \beta} \leq c\left(1+\frac{1}{1-\beta}\right)\left(1+\sup _{t \in K}\|t\|\right)
$$

and $c>0$ is an absolute constant. This implies the desired inequality of Proposition 1. 
Proof of Proposition 2. The inequality of Proposition 2 again easily follows from (i) of Theorem B. Indeed, for $0<\alpha<1 / 2$ we have

$$
\begin{aligned}
n^{1 / 2} c_{n}(\operatorname{cov}(K)) & \leq c_{K}\left(1+\sum_{k=1}^{n} k^{-1 / 2} e_{k}(K)\right) \\
& \leq c_{K}\left(1+\left(\sum_{k=1}^{n} k^{-1 / 2-\alpha}\right) \sup _{1 \leq k \leq n} k^{\alpha} e_{k}(K)\right) \\
& \leq c_{K}\left(1+\frac{1}{1 / 2-\alpha} n^{1 / 2-\alpha} \sup _{1 \leq k \leq n} k^{\alpha} e_{k}(K)\right),
\end{aligned}
$$

yielding, for all $n \in \mathbb{N}$,

$$
n^{\alpha} c_{n}(\operatorname{cov}(K)) \leq c_{K} \frac{2}{1-2 \alpha}\left(1+\sup _{1 \leq k \leq n} k^{\alpha} e_{n}(K)\right) .
$$

This estimate implies the inequality of Proposition 2.

Proof of Proposition 3. The estimates from above in the cases $0<\alpha<$ $1 / 2$ and $\alpha=1 / 2$ easily follow from (i) of Theorem B or from Propositions 1 and 2, whereas the estimate from above in the case $\alpha>1 / 2$ follows from (ii) of Theorem B. Now we show that the results are asymptotically optimal. The optimality in the case $0<\alpha<\infty$ and $\alpha \neq 1 / 2$ has already been proved in [CKP]. It remains to show the optimality in the critical case $\alpha=1 / 2$.

For this purpose we use the ingenious example of Gao [Ga] in the version of [CrSt]. Namely, there is a precompact subset $K$ of a Hilbert space $H$ such that for $n \in \mathbb{N}, e_{n}(K) \leq n^{-1 / 2} \log ^{-\beta}(n+1)$ and $e_{n}(\operatorname{cov}(K)) \geq$ $\gamma n^{-1 / 2} \log ^{1-\beta}(n+1)$, where $\gamma>0$ is an absolute constant. Fix $m \in \mathbb{N}$. Then from the inequality of Theorem $\mathrm{C}$ we get, with the sequence $s_{n}:=$ $n \log ^{\beta-1}(n+1)$,

$$
\begin{aligned}
& m n \log ^{\beta-1}(m n+1) e_{m n}(\operatorname{cov}(K)) \leq c_{\beta} \sup _{1 \leq k \leq m n} k \log ^{\beta-1}(k+1) c_{k}(\operatorname{cov}(K)) \\
& \leq c_{\beta}\left[\sup _{1 \leq k<n} k \log ^{\beta-1}(k+1) c_{k}(\operatorname{cov}(K))+\sup _{n \leq k \leq m n} k \log ^{\beta-1}(k+1) c_{k}(\operatorname{cov}(K))\right] .
\end{aligned}
$$

Inserting the entropy estimate $e_{k}(\operatorname{cov}(K))$ from below and the estimate of the Gelfand numbers $c_{k}(\operatorname{cov}(K)) \leq \alpha k^{-1 / 2} \log ^{1-\beta}(k+1)$ of Proposition 3 we arrive at

$$
\begin{aligned}
\gamma(m n)^{1 / 2} & \leq c_{\beta}\left(\alpha n^{1 / 2}+\sup _{1 \leq k \leq m n} k \log ^{\beta-1}(k+1) c_{k}(\operatorname{cov}(K))\right) \\
& \leq c_{\beta}\left(\alpha n^{1 / 2}+m n \log ^{\beta-1}(m n+1) c_{n}(\operatorname{cov}(K))\right) \\
& \leq c_{\beta}\left(\alpha n^{1 / 2}+m n \log ^{\beta-1}(n+1) c_{n}(\operatorname{cov}(K))\right)
\end{aligned}
$$

yielding

$$
\left(\gamma m^{1 / 2}-\alpha c_{\beta}\right) n^{1 / 2} \leq c_{\beta} m n \log ^{\beta-1}(n+1) c_{n}(\operatorname{cov}(K)) .
$$


Choose $m_{0}=m(\gamma, \alpha, \beta)$ such that $\gamma m_{0}^{1 / 2}-\alpha c_{\beta} \geq 1$. Then we get, with a positive constant $c_{\alpha, \beta, \gamma}$, the estimate

$$
c_{n}(\operatorname{cov}(K)) \geq c_{\alpha, \beta, \gamma} n^{-1 / 2} \log ^{1-\beta}(n+1) \quad \text { for } n \in \mathbb{N},
$$

which shows that the asymptotic behaviour of $c_{n}(\operatorname{cov}(K))$ is optimal.

Finally, we turn to the proof of Proposition 4. For this purpose we need an additional version of Pajor and Tomczak-Jaegermann's inequality. In order to formulate it we introduce the approximation numbers with respect to the $l$-norm. For an operator $T: E \rightarrow F$ between Banach spaces $E$ and $F$ the approximation numbers with respect to $l$ are defined by

$$
a_{n}(T ; l):=\inf \{l(T-A) \mid \operatorname{rank}(A)<n\}, \quad n \in \mathbb{N} .
$$

Lemma A. For an operator $T: E \rightarrow H$ from a Banach space $E$ into a Hilbert space $H$ we have the inequality

$$
k^{1 / 2} c_{k+n-1}(T) \leq \sqrt{2} a_{n}\left(T^{\prime} ; l\right) \quad \text { for } k, n \in \mathbb{N} .
$$

Proof. For the proof of this inequality we assume $l\left(T^{\prime}\right)<\infty$. Let $A$ : $H \rightarrow E^{\prime}$ be an operator with $\operatorname{rank}(A)<n$. Then

$$
c_{k+n-1}(T)=d_{k+n-1}\left(T^{\prime}\right) \leq d_{k}\left(T^{\prime}-A\right)+d_{n}(A)=d_{k}\left(T^{\prime}-A\right) .
$$

Thus by Theorem $\mathrm{D}$ we get

$$
k^{1 / 2} c_{k+n-1}(T) \leq k^{1 / 2} d_{k}\left(T^{\prime}-A\right) \leq \sqrt{2} l\left(T^{\prime}-A\right),
$$

and therefore,

$$
k^{1 / 2} c_{k+n-1}(T) \leq \sqrt{2} a_{n} l\left(T^{\prime}, l\right) \quad \text { for } k, n \in \mathbb{N} .
$$

Finally, we need the following result of Linde and Pietsch [LP]. In the following, $l_{\infty}$ as usual denotes the Banach space of all bounded sequences.

Lemma B. Let $D: l_{\infty} \rightarrow l_{\infty}, D\left(\xi_{i}\right)=\left(\sigma_{i} \xi_{i}\right)$, be a diagonal operator generated by a non-negative decreasing sequence $\sigma_{1} \geq \sigma_{2} \geq \ldots \geq 0$ such that $\sup \log ^{1 / 2}(n+1) \sigma_{n}<\infty$. Then there is a constant $c>0$ such that

$$
l(D) \leq c \sup _{n} \log ^{1 / 2}(n+1) \sigma_{n} .
$$

Proof of Proposition 4. It will be convenient to couch the arguments in the language of Gelfand numbers of operators. Indeed, if

$$
K=\left\{t_{1}, t_{2}, \ldots\right\} \subset H \quad \text { with }\left\|t_{n}\right\| \leq \sigma_{n}, \sigma_{1} \geq \sigma_{2} \geq \ldots \geq 0,
$$

and $\lim \sigma_{n}=0$, then we have

$$
c_{n}(\operatorname{cov}(K))=c_{n}\left(S D: l_{1}(\mathbb{N}) \rightarrow H\right),
$$

where the diagonal operator $D: l_{1} \rightarrow l_{1}$ is defined by $D\left(\xi_{i}\right)=\left(\sigma_{i} \xi_{i}\right)$, and $S: l_{1} \rightarrow H$ by $S e_{i}:=t_{i} / \sigma_{i}$ for $\sigma_{i}>0$ and $S e_{i}:=0$ for $\sigma_{i}=0$. 
Proof of (i). We factorize the operator $D$ as $D=D_{2} D_{1}$, where

$$
\begin{array}{ll}
D_{1}: l_{1} \rightarrow l_{1}, & \left(\xi_{i}\right) \mapsto\left(\log ^{1 / 2}(i+1) \sigma_{i} \xi_{i}\right), \\
D_{2}: l_{1} \rightarrow l_{1}, & \left(\xi_{i}\right) \mapsto\left(\log ^{-1 / 2}(i+1) \xi_{i}\right) .
\end{array}
$$

By Lemma 4 we get

$$
\begin{aligned}
n^{1 / 2} c_{2 n-1}\left(S D_{2} D_{1}\right) & \leq \sqrt{2} a_{n}\left(D_{1}^{\prime} D_{2}^{\prime} S^{\prime} ; l\right) \leq \sqrt{2} a_{n}\left(D_{1}^{\prime}\right) l\left(D_{2}^{\prime} S^{\prime}\right) \\
& \leq \sqrt{2} a_{n}\left(D_{1}\right) l\left(D_{2}^{\prime}\right)\left\|S^{\prime}\right\| \leq \sqrt{2} a_{n}\left(D_{1}\right) l\left(D_{2}^{\prime}\right)
\end{aligned}
$$

because of $\left\|S^{\prime}\right\|=\|S\| \leq 2$.

Since $\log ^{1 / 2}(n+1) \sigma_{n}$ is decreasing it follows that for all $n \in \mathbb{N}$,

$$
a_{n}\left(D_{1}\right) \leq \log ^{1 / 2}(n+1) \sigma_{n} .
$$

Moreover, by Lemma 6 we have

$$
l\left(D_{2}^{\prime}\right) \leq c
$$

Combining the previous estimates we obtain the desired estimate (i) of Proposition 4:

$$
n^{1 / 2} c_{2 n-1}(\operatorname{cov}(K))=n^{1 / 2} c_{2 n-1}(S D) \leq \sqrt{2} c \log ^{1 / 2}(n+1) \sigma_{n} .
$$

Proof of (ii). This time we decompose the operator $D: l_{1} \rightarrow l_{1}$ as

$$
D=D-D_{2^{n}}+D_{2^{n}}
$$

where

$$
D_{2^{n}}: l_{1} \rightarrow l_{1}, \quad\left(\xi_{i}\right) \mapsto \begin{cases}\sigma_{i} \xi_{i}, & i \leq 2^{n}, \\ 0, & i>2^{n} .\end{cases}
$$

Furthermore, we factorize $D_{2^{n}}$ as

$$
D_{2^{n}}=D_{2^{n}}^{(2)} D_{2^{n}}^{(1)},
$$

where

$$
\begin{array}{ll}
D_{2^{n}}^{(1)}: l_{1} \rightarrow l_{1}, & \left(\xi_{i}\right) \mapsto \begin{cases}\log ^{1 / 2}(i+1) \sigma_{i} \xi_{i}, & i \leq 2^{n}, \\
0, & i>2^{n},\end{cases} \\
D_{2^{n}}^{(2)}: l_{1} \rightarrow l_{1}, & \left(\xi_{i}\right) \mapsto \begin{cases}\log ^{-1 / 2}(i+1) \xi_{i}, & i \leq 2^{n}, \\
0, & i>2^{n} .\end{cases}
\end{array}
$$

Hence,

$$
\begin{aligned}
c_{n}(S D) & =c_{n}\left(S\left(D-D_{2^{n}}\right)+S D_{2^{n}}\right) \leq\|S\| \cdot\left\|D-D_{2^{n}}\right\|+c_{n}\left(S D_{2^{n}}\right) \\
& \leq \sigma_{2^{n}+1}+c_{n}\left(S D_{2^{n}}\right) \leq \sigma_{2^{n}}+c_{n}\left(S D_{2^{n}}\right)
\end{aligned}
$$

and

$$
\begin{aligned}
c_{n}\left(S D_{2^{n}}\right) & \leq c_{n}\left(S D_{2^{n}}^{(2)}\right)\left\|D_{2^{n}}^{(1)}\right\| \leq c_{n}\left(S D_{2^{n}}^{(2)}\right) \log ^{1 / 2}\left(2^{n}+1\right) \sigma_{2^{n}} \\
& \leq c_{n}\left(S D_{2^{n}}^{(2)}\right)(n+1)^{1 / 2} \sigma_{2^{n}} .
\end{aligned}
$$


Moreover, by Theorem D and Lemma 6 we get

$$
\begin{aligned}
n^{1 / 2} c_{n}\left(S D_{2^{n}}^{(2)}\right) & \leq \sqrt{2} l\left(\left(D_{2^{n}}^{(2)}\right)^{\prime} S^{\prime}\right) \leq \sqrt{2} l\left(\left(D_{2^{n}}^{(2)}\right)^{\prime}\right)\left\|S^{\prime}\right\| \\
& \leq \sqrt{2} c\|S\| \leq \sqrt{2} c .
\end{aligned}
$$

Combining the previous estimates we obtain the desired estimate:

$$
\begin{aligned}
c_{n}(\operatorname{cov}(K)) & =c_{n}(S D) \leq \sigma_{2^{n}}+c_{n}\left(S D_{2^{n}}\right) \\
& \leq \sigma_{2^{n}}+\sqrt{2} c\left(\frac{n+1}{n}\right)^{1 / 2} \sigma_{2^{n}} \leq(1+2 c) \sigma_{2^{n}} .
\end{aligned}
$$

The results are asymptotically optimal. Indeed, by Proposition 5.5 in $[\mathrm{CKP}]$ we deduce that for $K=\left\{\sigma_{n} e_{n}: n \in \mathbb{N}\right\} \subset l_{2}$, where $e_{n}$ is the unit vector basis of $l_{2}$ and $\sigma_{n}=\log ^{-\alpha}(n+1), 0<\alpha<\infty$,

$$
c_{n}(\operatorname{cov}(K)) \geq \begin{cases}c n^{-\alpha} & \text { for } 0<\alpha \leq 1 / 2, \\ c n^{-1 / 2} \log ^{1 / 2-\alpha}(n+1) & \text { for } \alpha>1 / 2 .\end{cases}
$$

\section{References}

[C1] B. Carl, Entropy numbers, s-numbers, and eigenvalue problems, J. Funct. Anal 41 (1981), 290-306.

[C2] - Metric entropy of convex hulls in Hilbert spaces, Bull. London Math. Soc. 29 (1997), 452-458.

[CKP] B. Carl, I. Kyrezi and A. Pajor, Metric entropy of convex hulls in Banach spaces, J. London Math. Soc. (2) 60 (1999), 871-896.

[CrSt] J. Creutzig and I. Steinwart, Metric entropy of convex hulls in type p spaces - the critical case, Proc. Amer. Math. Soc. 130 (2002), 733-743.

[Ga] F. Gao, Metric entropy of convex hulls, Israel J. Math. 123 (2001), 359-364.

[Go] Y. Gordon, On Milman's inequality and random subspaces which escape through a mesh in $\mathbb{R}^{n}$, in: Lecture Notes in Math. 1317, Springer, 1988, 84-106.

[LL] W. V. Li and W. Linde, Metric entropy of convex hulls in Hilbert spaces, Studia Math. 139 (2000), 29-45.

[LP] W. Linde and A. Pietsch, Mappings of Gaussian measures of cylindrical sets in Banach spaces, Teor. Veroyatnost. i Primenen. 19 (1974), 472-487 (in Russian).

[PT] A. Pajor and N. Tomczak-Jaegermann, Subspaces of small codimension of finite dimensional Banach spaces, Proc. Amer. Math. Soc. 97 (1986), 637-642.

[St] I. Steinwart, Entropy of $C(K)$-valued operators, J. Approx. Theory 103 (2000), 302-308.

Mathematisches Institut

Universität Jena

D-07740 Jena, Germany

E-mail: carl@minet.uni-jena.de
School of Mathematical Sciences University of Sussex Brighton BN1 9QH, UK E-mail: d.e.edmunds@sussex.ac.uk 